\title{
Lane Change Trajectory Prediction by using Recorded Human Driving Data
}

\author{
Wen Yao ${ }^{1}$, Huijing Zhao ${ }^{1}$, Philippe Bonnifait ${ }^{2}$, Hongbin Zha ${ }^{1}$
}

\begin{abstract}
Being able to predict the trajectory of a human driver's potential lane change behavior in urban high way scenario is crucial for lane change risk assessment task. A good prediction of the driver's lane change trajectory makes it possible to evaluate the risk and warn the driver beforehand. Rather than generating such a trajectory only using a mathematical model, this paper develops a lane change trajectory prediction approach based on real human driving data stored in a database. In real-time, the system generates parametric trajectories by interpolating $k$ human lane change trajectory instances from the pre-collected database that are similar to the current driving situation. In order to build this real lane change database, a human lane change data collection vehicle platform is developed. Extensive experiments have been carried out in urban highway environments to build a significant database with more than 200 lane changes. Real results show that this approach produces lane change trajectories that are quite similar to real ones which makes it suitable to predict humanlike lane change maneuvers.
\end{abstract}

\section{INTRODUCTION}

Past decades have witnessed tremendous progress in developing Advanced Driving Assistant Systems (ADAS) to improve safety. Such systems need to handle daily driving behaviors, among which lane change is a crucial issue. One meaningful application here is to pre-evaluate the potential risk before the driver decides to do a lane change or not, which can warn and prevent the driver from taking risky lane change behaviors beforehand. This application requires a prediction of the human driver's potential lane change trajectory for risk assessment. The key problem here is to generate a lane change trajectory which corresponds well to the driver's real lane change behavior.

Constant Yaw Rate and Acceleration (CYRA) model is commonly used to predict a trajectory and evaluate risks in near future. The CYRA model applies well in short time prediction (e.g $1000 \mathrm{~ms}$ ), but for long term behavior such as a whole lane change which usually lasts for $2 \sim 5 \mathrm{~s}$, as shown in Fig.1a, this model is far from accurate in such a complex driving scene, as it ignores a human driver's personal habits and the affects from the ego vehicle's environments. On the other hand, a trajectory could be predicted using a motion planning method as done in robotics.A set of candidates is generated on a parametric model and a trajectory is then selected optimizing an objective function which concerns

This work is partially supported by the NSFC-ANR Grants (No.61161130528), and the NSFC Grants (No.90920304).

${ }^{1}$ W. Yao, H. Zhao, and $H$. Zha are with the State Key Lab of Machine Perception (MOE), Peking University, Beijing, China. yao.wen@pku.edu.cn

${ }^{2} \mathrm{Ph}$. Bonnifait is with the Université de Technologie de Compiegne, Heudiasyc CNRS UMR 7253, France

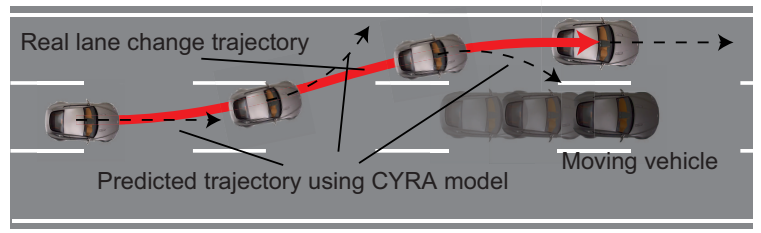

(a) CYRA model based trajectory prediction

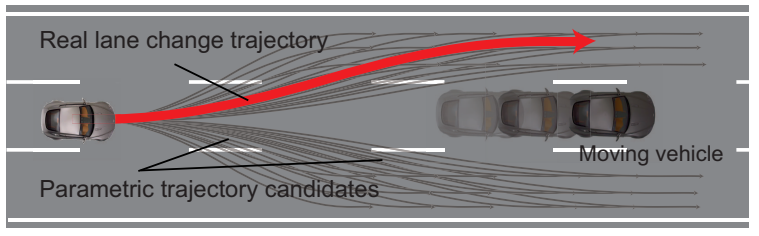

(b) Parametric lane change trajectory candidates

Fig. 1: Traditional trajectory prediction approach

parameters such as traveling distance, rate of acceleration (jerk), road constraints, interaction with other traffic participants, execution time or fuel consumption, etc. However, a human driver's decision making of a lane change maneuver in complex scene is far from being rigorously studied. It can have personal specificity, can vary during daily driving, and can be affected greatly by the local traffic situation.

This research proposes a trajectory prediction method for a potential lane change maneuver based on a set of human drivers' trajectory data that have been collected during real driving conditions with an instrumented vehicle on open roads. To this end, two sub-tasks are studied: 1) generation of a database that composes human drivers' trajectories during their lane change maneuvers on real road driving; 2) a trajectory prediction method based on real human driving database. The trajectory prediction method for risk estimation is evaluated by comparing with real lane change data.

Recent driving safety studies are becoming more and more focused on lane-level applications. The authors of [1] construct a road-map for highway lane change simulations in traffic simulation applications. The authors in [2] develop a parametric optimal trajectory generation method for dynamic highway scenarios that is able to plan for safe lane change and overtaking behaviors. A state lattice is used for trajectory generation in dynamic on-road driving scenarios in [3]. These algorithms are able to generate lane change trajectories that are free of collisions or that satisfy certain optimization objectives, such as minimizing jerk. Human drivers' characteristics are seldom considered in these studies. 


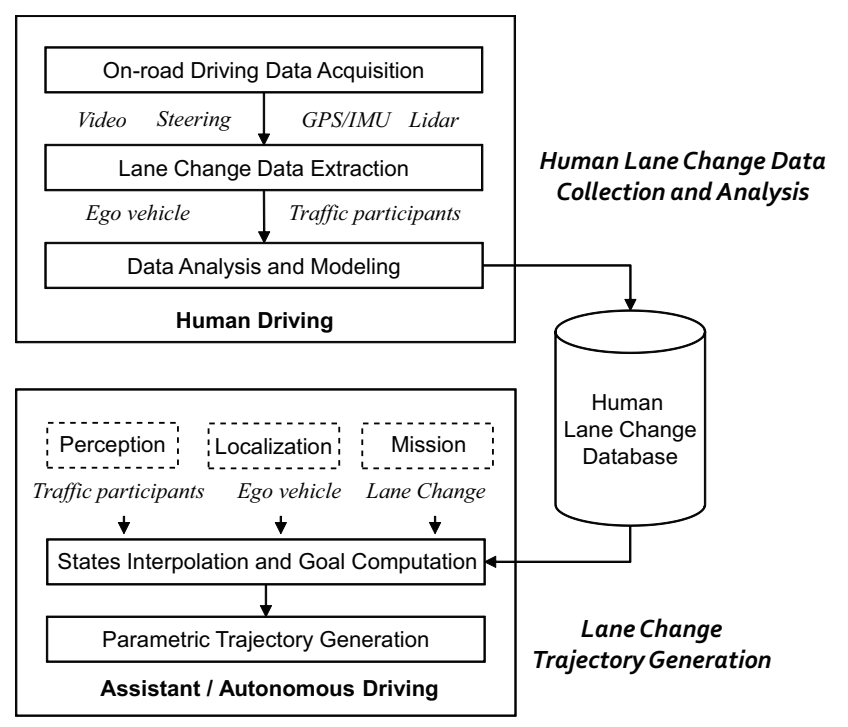

Fig. 2: Processing flow

There is a large amount of researches focused on human driver behavior. The researchers in [4] use differential GPS data to detect drivers' lane change behaviors. A steering model is built from a lane change behavior analysis in [5][6]. Our previous work extracted lane change trajectories from GPS data with the help of multi-modal sensors [7], but other traffic participants were not considered. Agents' preferred paths are learned dynamically and used to predict future states in [8][9]. Supervised learning approaches are used to learn driving behaviors for a car racing game in [10]. Similar research aiming at imitating human driving behaviors can be found in [11][12]. The effect of the surrounding traffic characteristics on lane-changing behavior is discussed in [13] with the goal of predicting the lane change motivation. To our best knowledge, few works try to predict lane change trajectories from real human lane change data.

The rest of this paper is organized as follows. Section 2 gives an overview of the lane change prediction system developed in this paper. Then, the lane change data collection process is introduced in Section 2. In Section 3, we develop an lane change trajectory prediction approach based on our human lane change database. Experimental results and evaluation are shown in Section 4. Conclusions and future work are described in Section 5.

\section{SYSTEM OVERVIEW}

This paper develops a lane change trajectory prediction approach for potential lane change behavior based on human driving data which is collected in urban highway environment. A parametric lane change trajectory is generated by referring to the k-nearest human driving instances in the database which are similar at their starting points with the present situation in online driving. Lane change trajectory predictions are only generated for lane change behaviors executing along straight roads which are the most common cases in urban highway scenario in Beijing, China, where the real driving data was collected. A lot of lane change

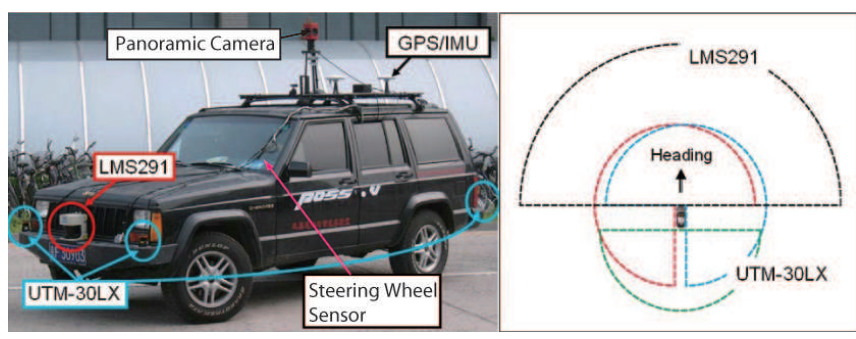

Fig. 3: Vehicle platform: POSS-V and sensor setting

trajectories can be recorded on the straight road in a single round along the city ring road. The data acquisition and analysis approach presented in this paper can be easily extended to lane change behaviors on curved road as long as the exact road shape is known as a prior knowledge.

As depicted in Fig.2, this work is divided into two parts: 1) human lane change data collection, and 2) lane change trajectory prediction based on human lane change data. In the first part, on-road experiments are conducted to collect human driving data using a test-bed vehicle with synchronized and calibrated multi-modal sensors. Data is processed to extract trajectories of the ego-vehicle during the lane changes, as well as those of other traffic participants nearby.

With these human driving data sets, the second part proposes a method for online lane change trajectory prediction. With the estimations of the ego and surrounding vehicles current states, which are supposed to be given by perception and localization modules, and with the goal to predict a lane change trajectory, the process looks for the K-nearest lane change instances in the human driving database which are similar at their starting states with the current driving situation. The end state is interpolated and used as the goal of the lane change maneuver. Subsequently, a trajectory that connects the start and end states is generated using existing trajecotry model.

\section{Data Collection of human lane Changes}

\section{A. On-road Driving Experiment and Data Acquisition}

An experimental vehicle has been manually driven in urban highway environment. Data collection experiments were carried out on the $4^{\text {th }}$ ring road of Beijing, China. It is one of the most important ring roads in Beijing with a uniform width and is mostly straight, so the road shape does not affect most of the lane change trajectories. The traffic was quite heavy but fluid enough during most of the experiments, without severe traffic jams that produce redundant data. Lane changes occurred quite often during the experiment at a speed of approximately $80 \mathrm{~km} / \mathrm{h}$ (which is the limited speed of the $4^{\text {th }}$ ring road with the length of $85.3 \mathrm{~km}$ ). We carried out several experiments and drove a total of 6 laps to collect more than 700 lane change trajectories. Our data collection platform POSS-V is shown in Fig. 3 left.

In this paper, to simultaneously record the lane change trajectory of the ego-vehicle and the position information of adjacent traffic participants, we combine two data collection systems that we used in prior works [7][14]. 


\section{B. Lane Change Trajectory Extraction for Ego-Vehicle}

For the ego-vehicle's lane change trajectory extraction, a GPS/IMU integrated system was used to record the global position and yaw angle with time stamps at a $10 \mathrm{~Hz}$ frequency. A small inertial measurement unit synchronized with the GPS was fixed on the steering wheel to record the driver's steering behavior. Panoramic camera recorded video information around the ego-vehicle and were used to visually check each lane change trajectory segmented from GPS and steering wheel data. The coarse start/end time of each lane change behavior is recorded manually, which makes possible to quickly locate the trajectory in GPS data. As discussed in [7], in a single round of driving on Ring Road 4, hundreds of lane change trajectories were recorded in the collected data.

\section{Trajectory Extraction for Traffic Participants}

For adjacent moving vehicles interacting with the egovehicle, we placed horizontal laser scanners to form omnidirectional coverage over a horizontal plane, as shown in Fig. 3 right. There were three low-cost Hokuyo UTM-30LXs, located at the front-left, front-right and middle-rear of the vehicle. They generated an omnidirectional horizontal coverage of the ego-vehicle's local vicinity with a measurement range normally up to $25 \mathrm{~m}$. A SICK LMS291 was additionally placed on the middle-front bumper to cover a semicircular area with a radius of up to $45 \mathrm{~m}$ in front of the ego-vehicle to provide better knowledge of the environment in front of the vehicle before and during lane changes. With these sensors, a moving object detection and tracking system has been developed in [14] to record the trajectories of the moving vehicles all around the ego-vehicle.

\section{LANE CHANGE TRAJECTORY PREDICTION USING HUMAN LANE CHANGE DATA}

Given the knowledge of the ego-vehicle state and the traffic participants nearby, we generate, in this section, a trajectory for an upcoming lane change. The state of current system under consideration is denoted $S=\left(S_{e g o}, S_{t p}\right)$. It is made up with the ego vehicle state $S_{\text {ego }}$ and the state $S_{t p}$ of the other participants. The components of these vectors are typically positions and speeds. The procedure here is to find a trajectory prediction function $F$ which maps the current system input state $x, x \in S$ to a predicted lane change trajectory $t, t \in T$, in the predicted lane change trajectory space $T$ :

$$
F: S \rightarrow T
$$

In this paper, the predicted lane change trajectory has a special need to be similar with personal driver's lane change behavior. As it is mentioned in section III, a set of $N$ known lane change "state-trajectory" data pairs $\left[\left(x_{1}, t_{1}\right),\left(x_{2}, t_{2}\right), \ldots\left(x_{N}, t_{N}\right)\right]$ were extracted from prerecorded human lane change behaviors building up a human lane change trajectory database $\Pi$. So, the prediction function $F$ which we seek for producing a predicted trajectory $t \in T$ for the driver in current situation $x \in S$ should satisfy:

$$
D_{\text {out }}\left(t, t_{i}\right)<\xi_{\text {out }}
$$

when

$$
D_{\text {in }}\left(x, x_{i}\right)<\xi_{\text {in }}
$$

meaning that when an input state $x$ comes, if there are some pre-recorded driving situations $x_{i}, i \in[1,2, \ldots N]$, which are quite similar to the input, with a similarity distance measure less then a small threshold $\xi_{i n}$, the predicted lane change trajectory $t$ should be no more different from the pre-recorded lane change trajectory $t_{i}$ than a certain small threshold $\xi_{\text {out }}$. The implementation procedure is: 1) To define the parameters describing the input driving situation $x$ and to find the most similar situations $\left\{x_{i}\right\}_{i=\{1,2 \ldots k\}}$ in the database $\Pi$ based on a certain similarity distance measure $D\left(x, x_{i}\right)$;2) To predict a lane change trajectory $t$ according to the pre-collected human lane change trajectories $\left\{t_{i}\right\}_{i=\{1,2, \ldots k\}}$ corresponding to these situations $\left\{x_{i}\right\}_{i=\{1,2 \ldots k\}}$ in the database. The detailed implementation of this work is as follows.

\section{A. Searching for similar input situations in the data base}

As we mentioned above, the current driving situation should include both the information from the ego-vehicle and the traffic participants around (or even some external parameters like road structure information if needed). The following parameters are available in our current sensor setting, being defined as the input state component:

$$
\begin{gathered}
S_{\text {ego }}=\left(C \cdot v_{H}, b\right), \quad b \in\{0,1\} \\
S_{t p}=\left(P_{F L}, P_{R L}, P_{F R}, P_{R R}, P_{F M}\right)
\end{gathered}
$$

Since the traffic participants that are crucial to lane change decisions should be the nearest ones in the following five directions around the ego-vehicle: Eq.5 includes the positions $P_{(\cdot)}=\left(x_{(\cdot)}, y_{(\cdot)}\right)$ of the front left $\left(M O_{F L}\right)$ and rear left $\left(M O_{R L}\right)$ ones for left lane changes, the front right $\left(M O_{F R}\right)$ and rear right $\left(M O_{R R}\right)$ ones for right lane changes, along with the front middle $\left(M O_{F M}\right)$ one for both maneuvers. Ego-vehicle speed $v_{H}$ is another important element to describe egovehicle's state. Other parameters such as vehicle acceleration and speed of surrounding vehicles can be easily added into the state vector if available. $C$ is a parameter which can be used to adjust the weight on $v_{H}$. A binary parameter $b$ indicates the current lane change behavior is a left or a right lane change. There may be no other traffic participants in one or more directions among the five directions mentioned above. In that case, we set their position in these directions to be a large constant $\left(X_{\max }, Y_{\max }\right)$ beyond the laser sensing range. The similarity distance measure $D\left(x, x^{\prime}\right)$ between the input situation $x$ and a giving situation $x^{\prime}$ in the database is defined as:.

$$
\begin{aligned}
D^{2}\left(x, x^{\prime}\right)= & b \cdot\left(\left\|P_{F R}-P_{F R}^{\prime}\right\|+\left\|P_{R R}-P_{R R}^{\prime}\right\|\right) \\
& +(1-b) \cdot\left(\left\|P_{F L}-P_{F L}^{\prime}\right\|+\left\|P_{R L}-P_{R L}^{\prime}\right\|\right) \\
& +\left\|P_{F M}-P_{F M}^{\prime}\right\|+C^{2} v_{H}^{2}
\end{aligned}
$$

when searching for the similar situations in the database, the distances between the input situation $x$ and each samples 


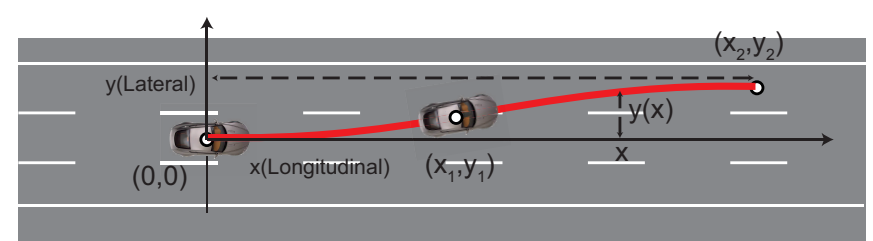

Fig. 4: Parametric trajectory generation approach

in the database are calculated and ranked. In this work, we just keep the first $k$ most similar situations $\left\{x_{i}\right\}_{i=\{1,2 \ldots k\}}$ satisfying Eq.3.

\section{B. Generating lane change trajectory based on collected lane change data}

Since the objective is to predict a lane change before it happens, a trajectory generation model is needed to transfer the trajectory prediction problem defined as Eq.1 to a two step procedure: The first step consists to estimate the parameters $p$ for the trajectory generation model in parameter space $P$. Function $f$ maps the input system state $S$ to the parameter states:

$$
f(x): x \rightarrow P, \quad x \in S
$$

The predicted trajectory $T$ for risk assessment is then derived through a trajectory generation model $\tau$ from this parameter $p$ :

$$
\tau(p): p \rightarrow T \quad p \in P
$$

Instead of simply applying a CYRA model, a parametric trajectory generation model in Frenet Frame [2] is selected to cooperate with the human lane change database mentioned above. As shown in Fig.4, the origin of the frame is the egovehicle position at the beginning of a lane change, with the $\mathrm{x}$-axis being along the direction of the current lane. Giving the predicted lane change end states as the control parameter $p$, the selected model produces a smooth trajectory from the origin. The following paragraph introduces the estimation of the parameter $p$ from the database and the details of the trajectory generation procedure:

1) Predicting the end states of the lane change for the current situation.: Giving the current driving situation $x \in S$, which includes both the ego-vehicle and traffic participants states, $k$ most similar lane change situations $\left\{x_{i}\right\}_{i \in\{1,2 \ldots k\}}$ are found in the database $\Pi$ based on the distance measured defined in Eq.6 and their corresponding lane change end states $\left\{p_{i}\right\}_{i \in\{1,2, \ldots k\}}$. Then, the predicted lane change end state $p$ can be interpolated from $\left\{p_{i}\right\}_{i \in\{1,2, \ldots k\}}$ for the current situation $x$ using Inverse Distance Weights:

$$
p=f(x)=\sum_{\delta \subseteq \Pi} \frac{\omega_{i}(x) p_{i}}{\sum_{\delta} \omega_{j}(x)}
$$

where

$$
\omega_{i}=\frac{\frac{1}{D\left(x_{e}, x_{j}\right)^{2}}}{\sum_{j \in \delta} \frac{1}{D\left(x_{e}, x_{j}\right)^{2}}}
$$

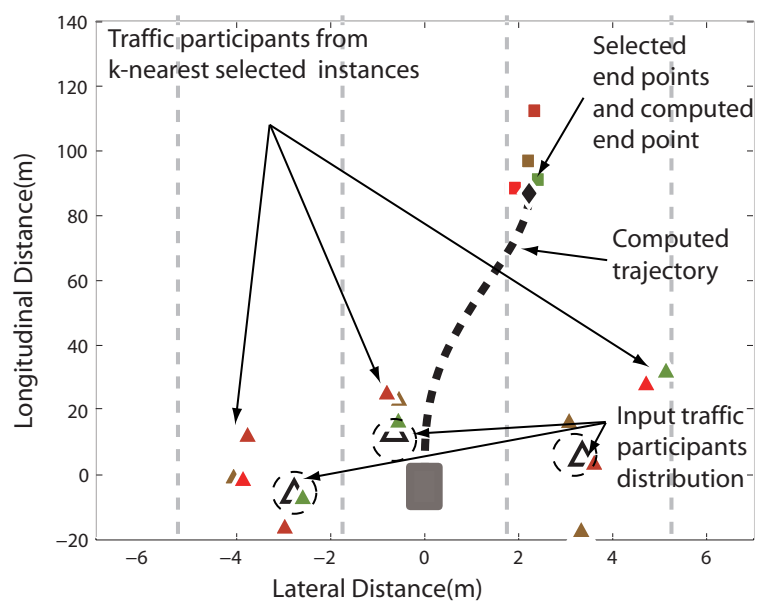

Fig. 5: K most similar traffic participants' distribution (color solid triangle) with generated (black dash curve) and real lane change trajectory (green dash curve)

The $k$ nearest instances are found according to $v_{H}$ if there is no traffic participant in the sensing range. For a given input ego-vehicle situation $x_{e}$ represented in such a state vector, we find the $k$ most similar instances $\delta$ as defined above with their trajectories ending in the ego-frame. Here, $D_{e j}$ is the distance measure defined in Eq.6 between the selected $j_{t h}$ instance and the ego-vehicle at the input situation. This interpolation approach puts higher weight on instances with smaller distance which encourages the lane change maneuvers happened in similar driving situations to have more effect on the result. The end state interpolation procedure is shown in Fig. 5, where solid triangles represent the traffic participants' position of the $k(k=4$ in our experiment) nearest instances with different colors. The black triangles are the adjacent traffic participants' position of current input. The colored squares are the end points of the selected instances. The black diamond is the end point interpolated from these end points. The black dashed curve is a parametric trajectory generated, which will be introduced below.

2) Parametric trajectory generation model : In our data set, no detailed road information is currently available. Remember that the origin of the frame is the ego-vehicle position at the beginning of a lane change and the $\mathrm{x}$-axis to be along the direction of the current lane, as shown in Fig. 4. By considering the following polynomial curve

$$
y(x)=a_{0}+a_{1} x+a_{2} x^{2}+a_{3} x^{3}+a_{4} x^{4}+a_{5} x^{5}
$$

which satisfies

$$
\left\{\begin{array}{l}
y(0)=0 \\
y\left(x_{2}\right)=y_{2} \\
\frac{d y}{d x}(0)=\frac{d y}{d x}\left(x_{2}\right)=0 \\
\frac{d^{2} y}{d x^{2}}(0)=\frac{d^{2} y}{d x^{2}}\left(x_{2}\right)=0
\end{array}\right.
$$


with the end states $\left(x_{2}, y_{2}\right)$ computed as in Section 3.A, one can obtain a path represented with the six parameters $\left\{a_{i}\right\}_{i=0 \sim 5}$ by easily solving a linear system for the 3 nonzero parameters in this particular case. The result curve has no lateral speed or acceleration at $\left(x_{2}, y_{2}\right)$ where a lane change maneuver finishes. Please notice that the obtained curve is a Taylor's expansion of clothoid up to the fifth order, which meets the non-holonomic constrains for motionless car-like vehicles.

Currently, in the data base we have collected, it is difficult to obtain the precise acceleration of the ego-vehicle, so we define a speed profile along this path. It can be chosen, for instance, to keep constant the initial value of the vehicle speed, or it is possible to compute a constant acceleration to reach the weighted sum of the average velocity in the $k$ nearest lane change trajectory instances. In our experiments, we keep ego-vehicle's speed at the beginning of the lane change as the speed profile.

\section{EXPERIMENTAL RESULTS}

Since there is large amount of data, to clearly show the data features, only the data from the most recent experiment data is displayed on Fig. 6. The driver achieved lane change behavior according to his driving habit during the experiment. 236 lane changes and more than 1000 adjacent traffic participants' trajectories were recorded. A moving local frame fixed on the ego-vehicle with $y$-axis always parallel to the road direction (Fig. 6a left) is used to visualize the collected lane change data. Fig. 6a right shows all the traffic participants' position at the beginning of each lane change of the ego-vehicle. Though we have no lane detection sensors in our system, the distribution of traffic participants along the lateral axis shows distinct sets which corresponds to traffic lanes. The lane width of Ring Road 4 is $3.5 \mathrm{~m}$ which supports the result shown in the figure. Fig. $6 \mathrm{~b}$ to Fig. $6 \mathrm{~d}$ show left lane change data in the left column and right lane change data in the right, as sketched in Fig. 6b;

\section{A. Traffic participants' trajectories in a lane change}

Traffic participants' trajectories in a lane change maneuver include the information of lane change direction and relative speed of adjacent vehicles. Fig. 6c left depicts a maneuver involving the ego-vehicle (Grey rectangle in the middle) merging into the left lane between two vehicles (marked as $M O_{F L}$ and $M O_{R L}$ in the figure). Adjacent vehicles move from green arrows $\left(x_{s}, y_{s}\right)$ to the other end of the trajectory in this ego-vehicle's local frame. It is obvious that if an adjacent vehicle is not changing lanes in the same direction as the ego-vehicle, it should end at $\left(x_{e}, y_{e}\right)$, which satisfies $x_{e}<x_{s}$ for a left lane change when the maneuver finishes. We also know that $y_{e}>y_{s}$ means that the moving object is driving at a higher speed than the ego-vehicle and vice versa. Fig. $6 \mathrm{c}$ right shows the ego-vehicle change to the right lane to follow $M O_{F R}$ to overtake $M O_{F M}$ in front.
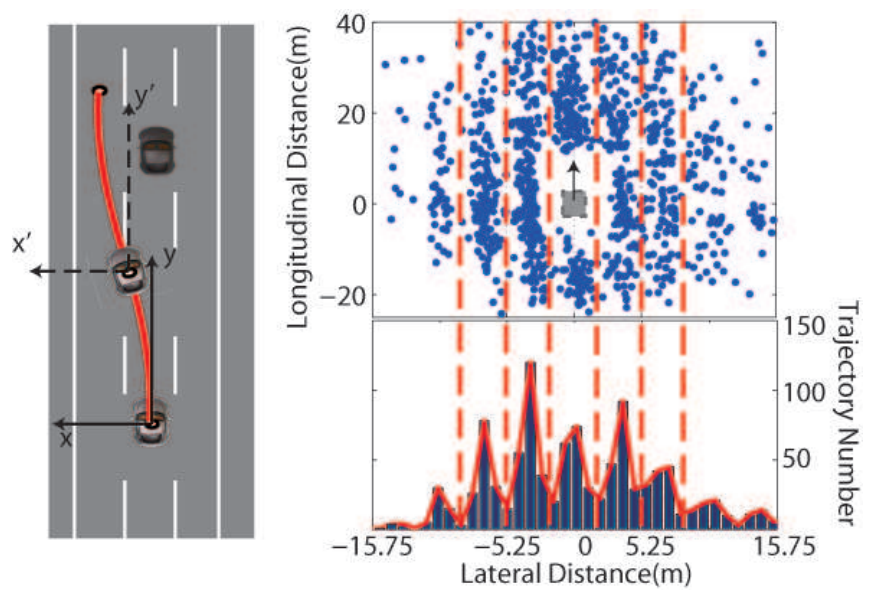

(a) A moving local frame (left) in which traffic participants distribution implies road lanes (right)
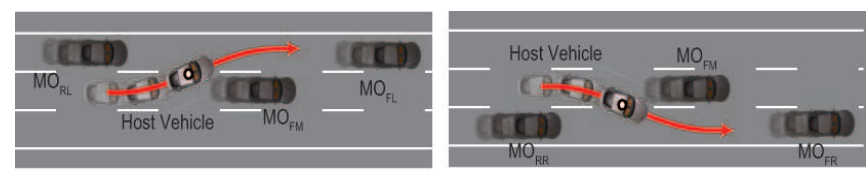

(b) Typical lane change trajectory (to left/right lane)
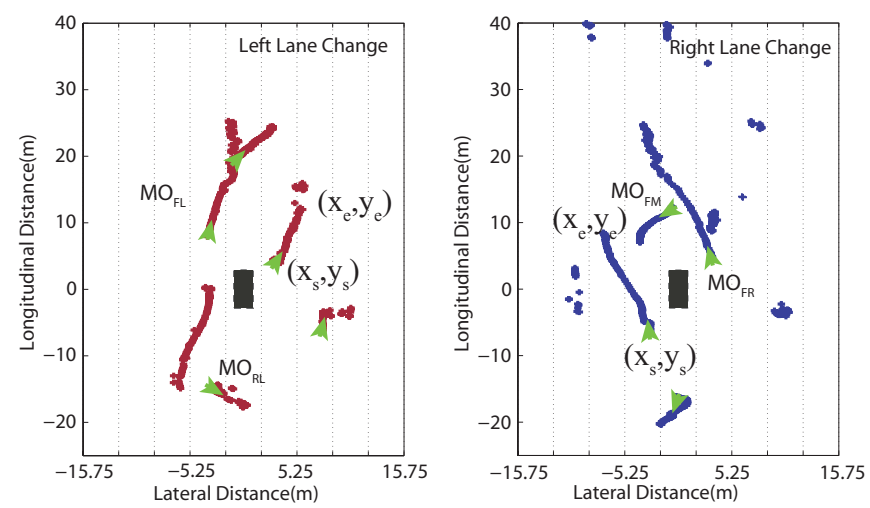

(c) Adjacent traffic participants' relative position in one lane change maneuver
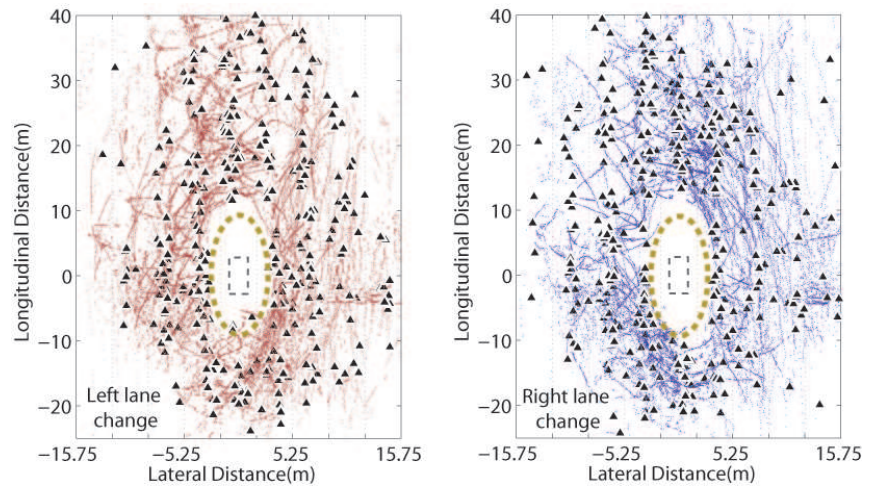

(d) Adjacent traffic participants' relative position during whole experiment

Fig. 6: Adjacent traffic participants' relative position data in local coordinate when the ego-vehicle is in lane change maneuver

\section{B. Visualization of traffic participants' trajectories in all lane change}

The trajectories of the other traffic participants during every complete lane change are drawn in Fig. 6d. It is 
important to note that there is an area much larger than the vehicle size around the ego-vehicle in the figure. This area is almost free of any moving obstacles, as indicated by an ellipse in Fig. 6d. This area can be regarded as the safety zone required during lane change maneuvers. It shows the lower safety bound that the driver maintains relative to other vehicles in all directions when performing lane changes in current driving situations, at a speed of approximately $80 \mathrm{~km} / \mathrm{h}$ on the ring road in our experiment. For convenience, an ellipse is used to represent this obstaclefree area conservatively, which will be used to check and evaluate the trajectory generation results in the following subsection.

\section{Trajectory prediction result evaluation}

To evaluate the trajectory prediction results, trajectories are randomly excluded from the data base as test instances. The ego-vehicle's real lane change trajectory and traffic participants' movements are replayed in the same time scale with a virtual ego-vehicle moving along the predicted trajectory. Fig. 8a shows a $5 s$ lane change maneuver in a simple situation where only 2 traffic participants (red circle) appeared near the ego-vehicle. It can be seen that the Grey car (virtual ego-vehicle) moving along the generated trajectory (black) keeps quite close to the red car (real ego-vehicle) along the real trajectory recorded. The traffic participants' positions in the ego-vehicle's local frame are shown on the right, where the red dotted ellipse $x^{2} / 9+y^{2} / 64=1$ represents the "obstacle-free" area (or safety zone) as we mentioned in Section 3. The blue "+"s are the traffic participants' relative position to the virtual ego-vehicle and the green "+"s are real data. Fig. 8 b depicts a more complex situation where 5 adjacent traffic participants appeared, but all traffic participants' trajectory in local frame keep out of the obstacle-free area, which means no collision will happen if the ego-vehicle executes the predicted trajectory.

To prove the reliability of the method, we test all of the collected trajectories and draw the traffic participants' movement in the local frame both for real (right) and generated trajectories (left) in Fig. 7. It is clear that most traffic participants remain outside of the "obstacle-free" area and have similar distributions with real data near the egovehicle, which means the the situation when human drivers executed a lane change is mostly predicted still as safe for lane change and the predicted behavior is similar with the way the human driver does. However, it may judge some situation in which human driver actually did a lane change to be risky (e.g., traffic participants' position appeared in "obstacle-free area" in 7 left). We found 3 real lane change situations out of a total 236 lane change behavior, being evaluated as risky situation. Meaning that the predicted lane change trajectory will lead to the situation where obstacles would appear in the highlighted ellipse area (the "obstaclefree" area). This is due to the changing of driving situation during lane changes is not completely predictable only based on the initial states and the current data base may be not big enough. Further experiments are needed to improve the
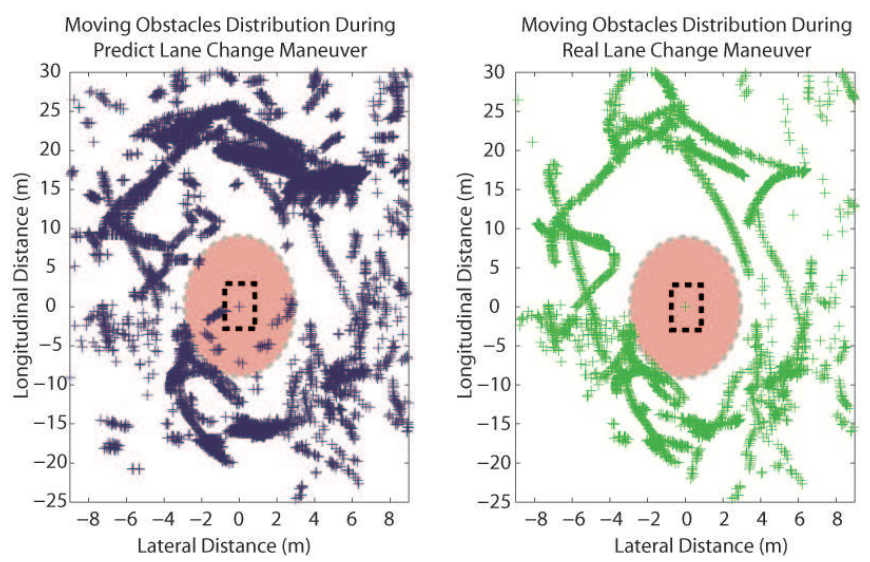

Fig. 7: Traffic participants' position in the ego-vehicle's local frame when the ego-vehicle executes real trajectories (right) and generated trajectories (left).

database and validate the proper size of database which may give acceptable prediction results for lane change prediction while keeping the efficiency.

\section{CONCLUSION AND FUTURE WORK}

This research aims at predicting lane change trajectories based on real human lane change data. A system combining lane change trajectory extraction and moving obstacle detection and tracking is used to collect on-road human lane change information. An parametric lane change trajectory prediction approach is developed by finding the $k$ nearest real lane change instances and generating a similar parametric trajectory according to these instances. The experimental results show that our approach can produce human-like lane change trajectory prediction results which are similar to human driver. Future work will focus on the current limitations of the approach: increasing the lane change database while keeping efficiency. It is also planed to compare with existing mathematical trajectory generation algorithms on real data to show the relevance of producing human-like trajectories using our approach. Additionally, it will be interesting to include lane change data from different drivers and different driving environments for driving style comparison.

\section{REFERENCES}

[1] Jur van den Berg, J. Sewall, M.Lin, D. Manocha, Virtualized Traffic: reconstructing traffic flows from discrete spatio-temporal data, Virtual Reality Conf., Lafayette, LA, Mar 2009, pp. 183-190.

[2] M. Werling, J.Ziegler, S. Kammel, S. Thrun, Optimal trajectory generation for dynamic street scenarios in a Frenet Frame, IEEE Int. Conf. on Robotics and Automation, Anchorage, AK, May 2010g, pp. 987-993.

[3] J. Ziegler and C. Stiller, Spatiotemporal state lattices for fast trajectory planning in dynamic on-road driving scenarios, IEEE/RSJ Int. Conf. on Intelligent Robots and Systems, St. Louis, 2009, pp. 1879-1884.

[4] Y. Xuan and B. Coifman, Lane change maneuver detection from probe vehicle DGPS data, IEEE Intelligent Transportation Systems Conference, Toronto, Ont, Sept 2006, pp. 624-629.

[5] R. Hess and A. Modjtahedzadeh, A control theoretic model of driver steering behavior, IEEE Control Systems Magazine, 1990, pp. 3-8.

[6] J. Feng, J. Ruan and Y. Li, Study on intelligent vehicle lane change path planning and control simulation, IEEE Int. Conf. on Information Acquisition, Shandong, Aug 2006, pp. 683-688. 

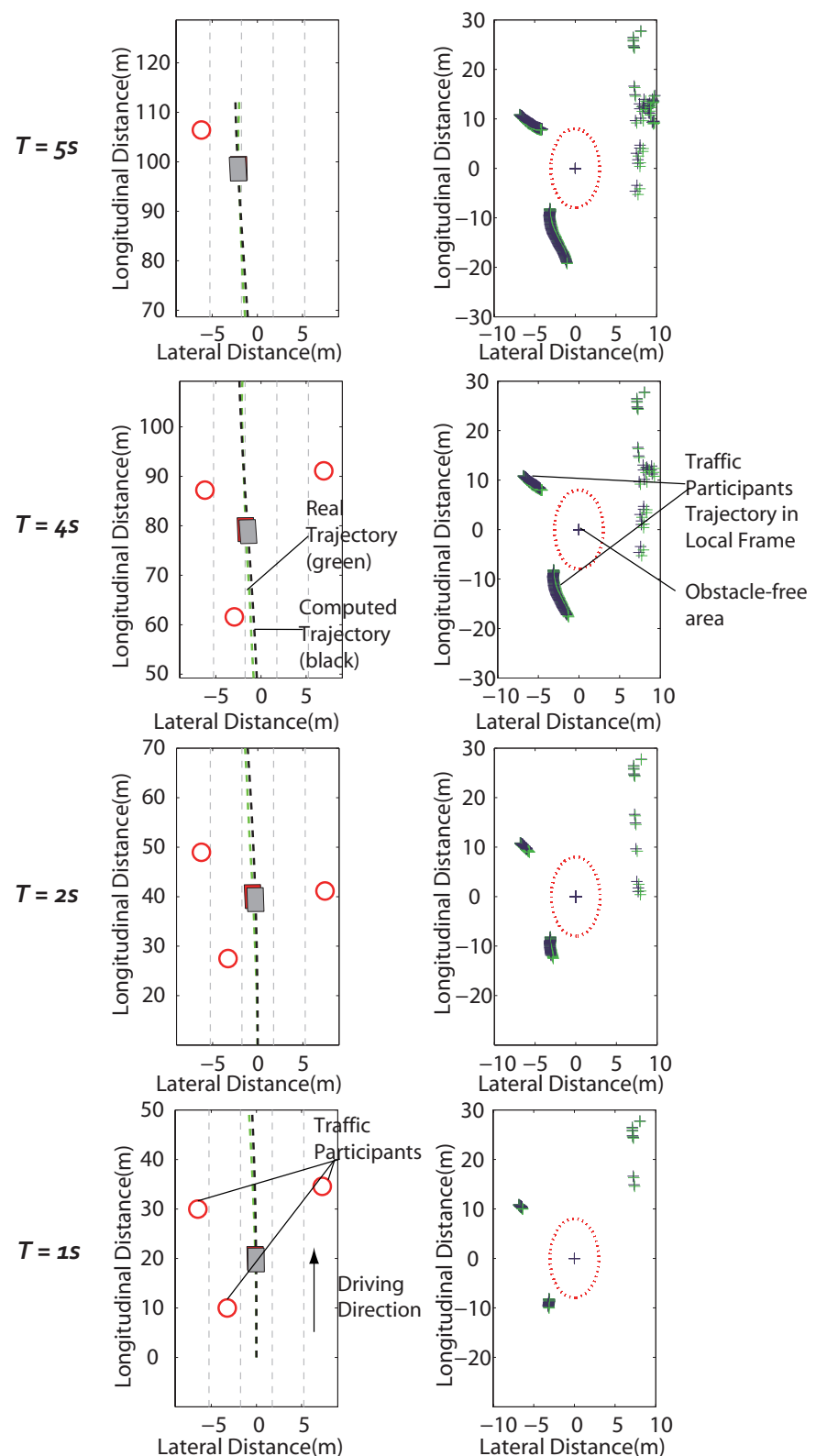

(a) Trajectory generation to left lane
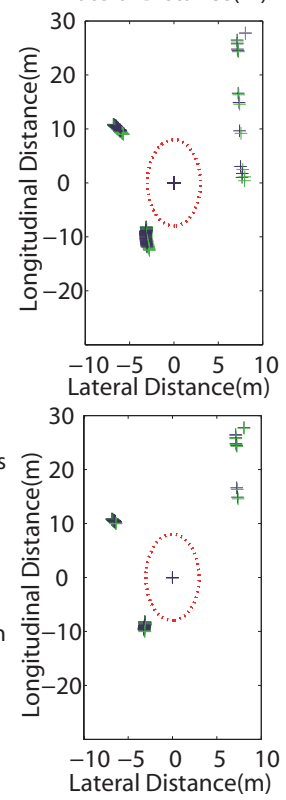
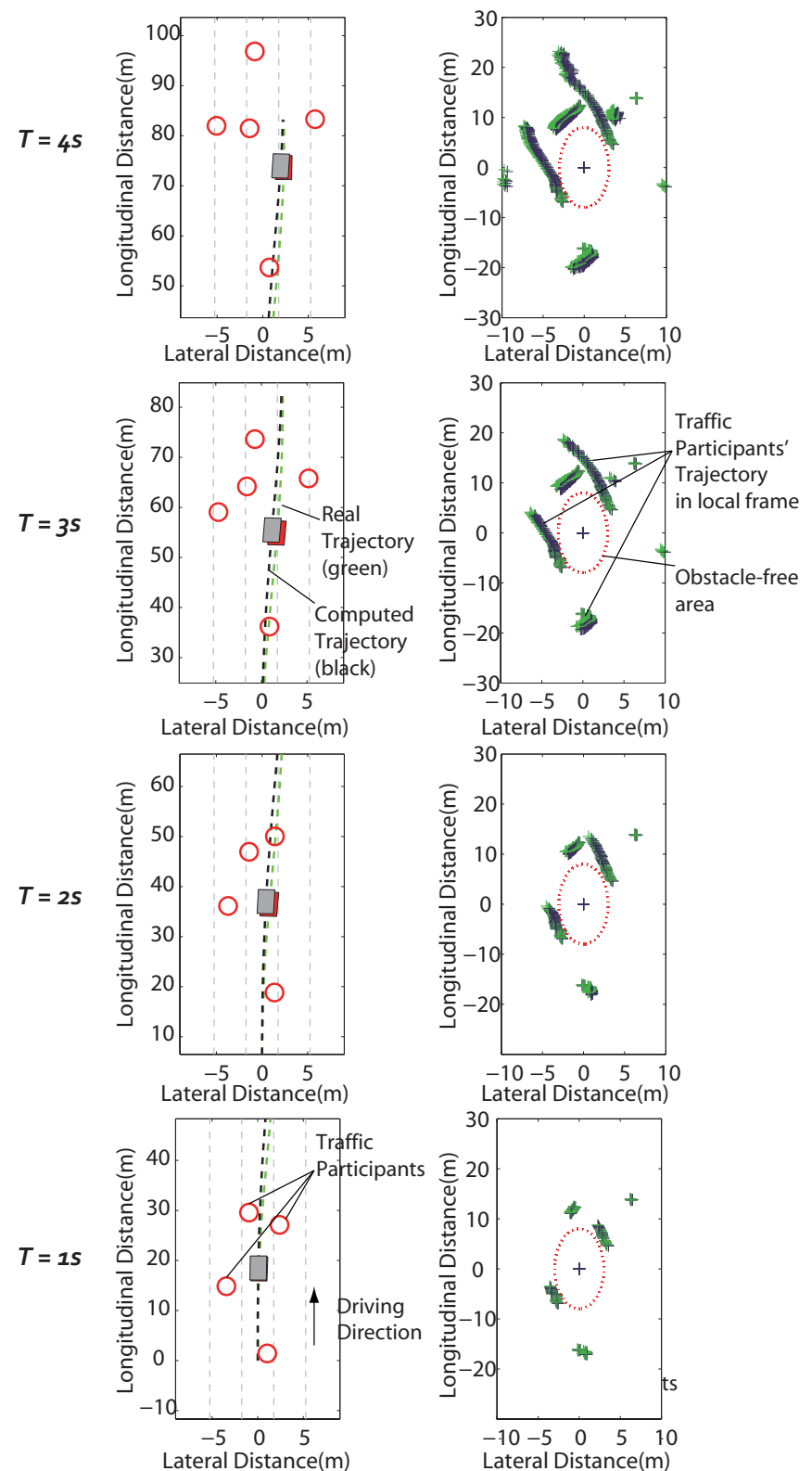

(b) Trajectory generation to right lane

Fig. 8: Comparison of real lane change trajectories with computed maneuvers

[7] W. Yao, H. Zhao, F. Davoine, H. Zha, Learning Lane change trajectories from on-road driving data, IEEE Intelligent Vehicles Symposium, Alcala de Henares, June 2012, pp. 885-890

[8] H. Helble and S. Cameron, 3-d path planning and target trajectory prediction for the oxford aerial tracking system, in IEEE Int. Conf. on Robotics and Automation, April 2007, pp. 1042“C1048.

[9] M. Bennewitz, W. Burgard, G. Cielniak, and S. Thrun, Learning motion patterns of people for compliant robot motion, Int. Journal of Robotics Research, vol. 24, pp. 31“C48, 2005.

[10] L. Cardamone, D. Loiacono and P. L. Lanzi, Learning drivers for TORCS through imitation using supervised methods, IEEE Symp. on Computational Intelligence and Games, 2009, pp. 148-155.

[11] P. Abbeel, A. Y. Ng, Apprenticeship learning via inverse reinforcement learning, Proceedings of the 21st Int. Conf. on Machine Learning,
Banff, Canada, 2004.

[12] D. Loiacono, A. Prete, P. L. Lanzi, L. Cardamone, Learning to overtake in TORCS using simple reinforcement learning, IEEE Congress on Evolutionary Computation (CEC), Barcelona, July 2010, pp. 1-8.

[13] S. Moridpour, G. Rose, and M. Sarvi, Effect of surrounding traffic characteristics on lane changing behavior, Journal of Transportation Engineering, vol. 136, pp.973-985. 2010.

[14] H. Zhao, C. Wang, W. Yao, F. Davoine, J. Cui, H. Zha, Omnidirectional detection and tracking of on-road vehicles using multiple horizontal laser scanners, IEEE Intelligent Vehicles Symposium, Alcala de Henares, June 2012, pp. 57-62.

[15] A. Kelly, B. Nagy, Reactive nonholonomic trajectory generation via parametric optimal control, Int. Journal of Robotics Research, vol. 22, pp. 583-601, July 2003. 